\title{
Research on the Correlation of Control Malfunction with Induced Voltage of Control Signal Line According to Voltage Change of a Power Line
}

\author{
Dong-Woo Kang*, In-Gun Kim**, Sang-Hwan Ham***, Sung-Yul Kim*, Sungwoo Bae ${ }^{\S}$, \\ Dae-Nyeon Kim ${ }^{\S \S}$ and Ju Lee ${ }^{\dagger}$
}

\begin{abstract}
This induced voltage measurement test and electromagnetic field simulation are related to the possibility of control signal malfunction by power line. Through an experiment, this research analyzed whether the voltage causing control malfunction according to the on/off status of power permitted to power line was induced to control signal line. Also, the research calculated the voltage induced to control signal line and examined the phenomenon by conducting an electro-magnetic fieldspecific simulation through the finite element method for the cable model used in the experiment.
\end{abstract}

Keywords: Control signal line, Induced voltage, Control malfunction, Overall + individual shield ground

\section{Introduction}

As the voltage induced in power line can cause control signal malfunctions when there exist both the power line and control signal line for propelling the system, this paper states that preparation against such problems is needed. $[1,2]$

To measure whether the voltage causing control signal malfunction is induced by a nearby power line, a test was performed by preparing an integrated cable sample, which was then modeled to analyze the phenomenon through an electromagnetic field characteristics simulation. The cable used in the experiment was ' $0.6 / 1 \mathrm{kV}$ TFR-CVVS, $8 \mathrm{P} \times$ $1.5 \mathrm{~mm}^{2}$ cable (hereinafter integrated cable)' from LS Wire, and the voltage induced to the control signal line based on the on/off status of power line was measured.

\section{Body}

2.1 Measurement of control signal line induced voltage

The test circuit was connected as in Fig. 1, and the cable

$\dagger$ Corresponding Author: Dept. of Electrical Engineering, Hanyang University, Korea. (julee@hanyang.ac.kr)

* Dept. of Electrical Energy Engineering, Keimyung University, Korea. (dwkang@kmu.ac.kr)

** Dept. of Electrical Engineering, Hanyang University, Korea (waterkig@naver.com)

*** School of Electrical and Railway Engineering, Kyungil University, Korea. (shham@kiu.kr)

$\S$ Dept. of Electrical Engineering, Yeungnam University, Korea (sbae@yu.ac.kr)

$\S \S \quad$ Research Equipment Technology Team, Kyeongbuk Institute of IT Convergence Industry Technology, Korea. (nyeonny@gmail.com)

Received: October 22, 2015; Accepted: January 23, 2016 was arranged as in Fig. 2. Then the control signal line was connected to the terminal block inside the terminal box to minimize the contact elements among stranded cables. In

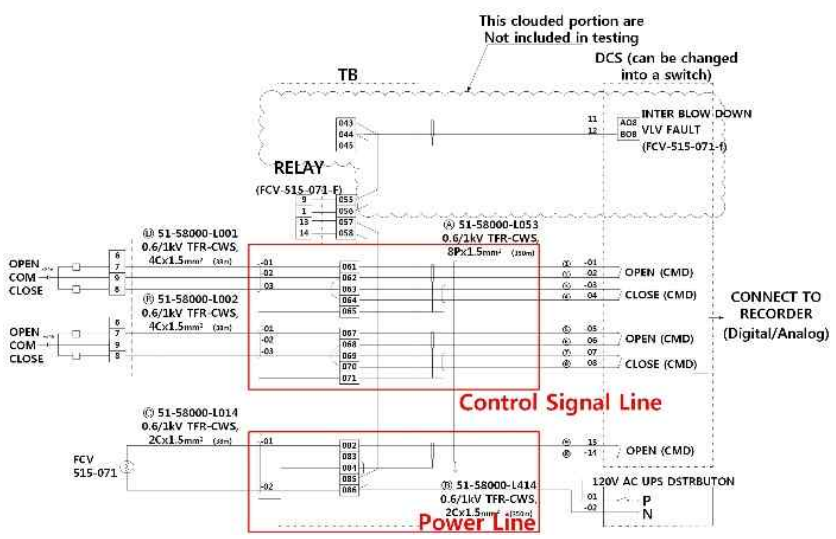

Fig. 1. Test circuit diagram

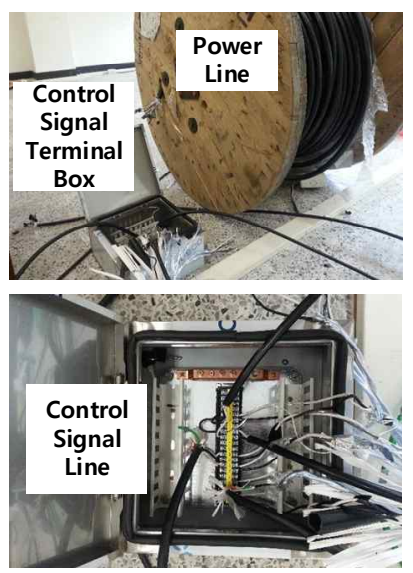

Fig. 2. Test measurement cable and terminal box 
accordance with the integrated cable ground status, the voltage induced to the control signal line was expected to be difficult. Thus the ground state was divided into overall shield ground (experiment 1) and overall + individual shield ground (experiment 2) to conduct the test. [3, 4]

\subsection{Analysis and review of test result}

The integrated cable consists of a total of 8 pairs and 5 pairs among them were used. 1 pair was the power line and 4 pairs were used as control signal lines to send signals.

This test permitted $120 \mathrm{Vac}(60 \mathrm{~Hz})$ phase voltage, a driving voltage, on the power line through UPS by minimizing the noise of power supply terminal and measured the changes of voltage on the 4 pairs of signal lines adjacent to the power line. Lecroy's Wave Runner 610Zi model was used as a measurement tool for sampling, and CP0303 current probe and HVD3102 voltage differential probe were used for measurement.

The switch for power line on/off was switched at an interval of about 1.5 seconds. The graph measured with an oscilloscope was in the order of voltage, electric current and partial enlargement of voltage at on/off of the control signal line. When the overall shield ground was used, the measured induced voltage was as in Fig. 3. The maximum

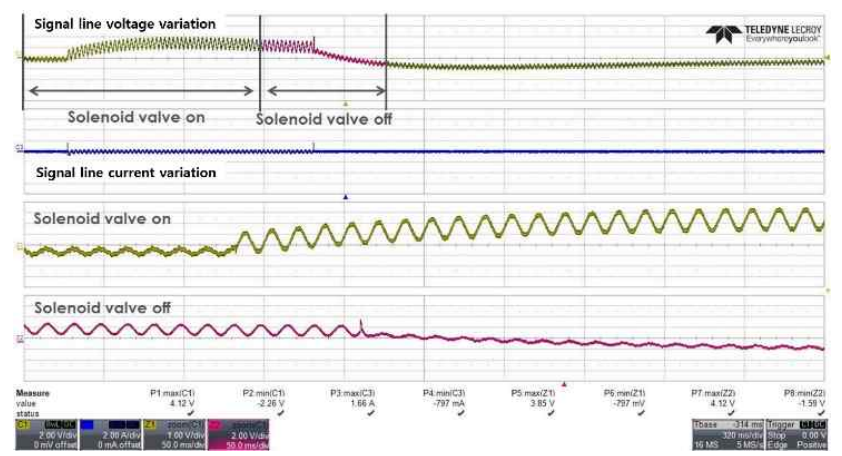

Fig. 3. The induced voltage of signal line measured with an oscilloscope during power on/off at an overall shield ground state

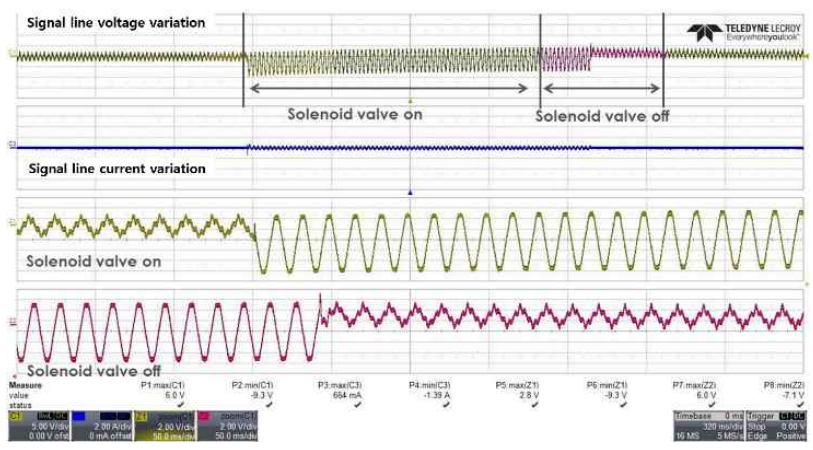

Fig. 4. The induced voltage of signal line measured with an oscilloscope during power on/off at an overall + individual shield ground state voltage measured in this test was $4.8 \mathrm{~V}$ at power on and 5.9 at power off, and the voltage was measured over 100 times for deviation analysis.

The test result after dividing the ground state into overall shield ground and overall + individual shield ground was as in Fig. 4. The voltage induced by using overall + individual shield ground was measured to be a maximum of $13.5 \mathrm{~V}$ at power on and a maximum of 15.1 at power off. As seen on the result, 1.6V higher voltage at power off was induced to the control signal line, and its deviation analysis result was represented on Fig. 5 and 6 .

A summary of test result was shown on Table 1. If the control signal line and power line were in the same integrated cable, the variation of magnetic flux according to the voltage change of power line abandoned certain voltage on the control signal line. Such phenomenon has the same principle as the $1: 1$ transformer of $1 / 2$ turn as seen on Fig. 7 below. $V_{1}$ is power line voltage and its

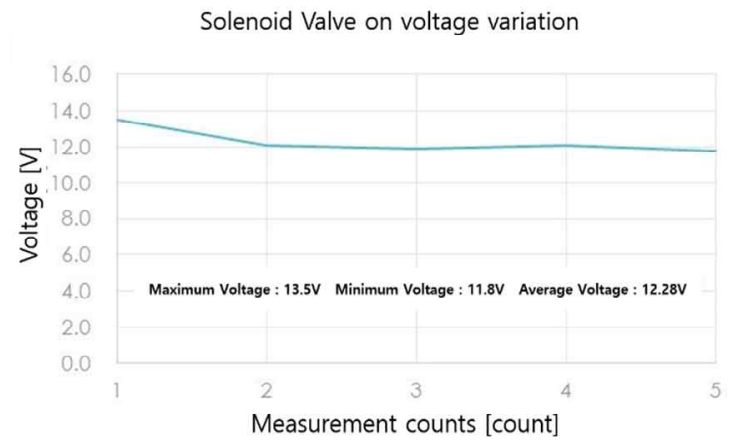

Fig. 5. Deviation analysis on the induced voltage of signal line measured by oscilloscope at power on during overall + individual shield ground state power

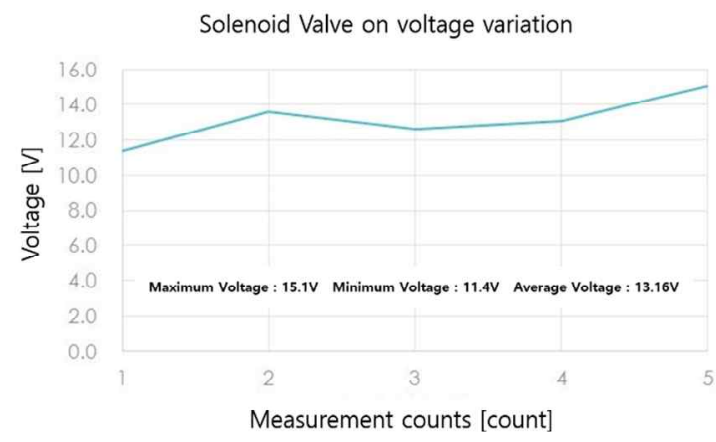

Fig. 6. Deviation analysis on the induced voltage of signal line measured by oscilloscope at power off during overall + individual shield ground state power

Table 1. Test result of voltage induced by integrated cable

\begin{tabular}{c|c|c|c|c}
\hline Distribution & & \multicolumn{3}{|c}{ Voltage Variation } \\
\hline Ground Situation & Switch Situation & Max & Min & Avg \\
\hline \multirow{2}{*}{ Overall shield } & Switch on & $4.8 \mathrm{~V}$ & $2.3 \mathrm{~V}$ & $3.6 \mathrm{~V}$ \\
\cline { 2 - 5 } & Switch off & $5.9 \mathrm{~V}$ & $1.7 \mathrm{~V}$ & $3.8 \mathrm{~V}$ \\
\hline $\begin{array}{c}\text { Overall + individual } \\
\text { shield }\end{array}$ & Switch on & $13.5 \mathrm{~V}$ & $11.8 \mathrm{~V}$ & $12.28 \mathrm{~V}$ \\
\cline { 2 - 5 } & Switch off & $15.1 \mathrm{~V}$ & $11.4 \mathrm{~V}$ & $13.16 \mathrm{~V}$ \\
\hline
\end{tabular}




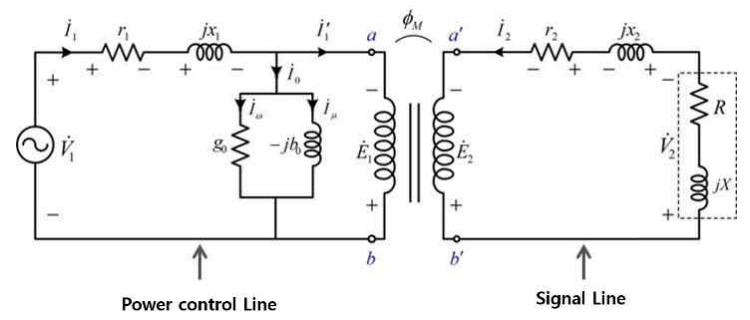

Fig. 7. Equivalent circuit of 1:1 transformer [7]

alternating current is $120 \mathrm{Vac}$, and $\mathrm{r}_{1}$ has a very value as the resistance of power line. $V_{2}$ is load voltage and $R, X$ is load impedance. $\mathrm{E}_{1}, \mathrm{E}_{2}$ is primary, secondary induced voltage. $\mathrm{I}_{1}$ is power line current, $\mathrm{I}_{\mathrm{o}}$ is exciting current, $\mathrm{I} \omega$ is Iron loss current and $I \mu$ is magnetizing current. $I_{1}{ }_{1}$ is primary load current. Leakage reactance $\mathrm{x}_{1}$, which represents leakage, is determined by the separation distance between power line and signal line, and if there is no leakage $(100 \%$ magnetic coupling) $120 \mathrm{~V}$ can be abandoned to the control signal line. However, as the insulator inside the cable has the relative permeability of 1 , a huge leakage flux is expected to occur and a low voltage is abandoned to the control signal line as shown on the actual test. $[5,6]$

If there is an enough separation distance, the leakage reactance can be very big ( $0 \%$ magnetic coupling) and voltage may not be abandoned to the control signal line. However, according to the cross-sectional diagram of the cable, there is no separation distance and the leakage reactance is considered for control signal line, so certain voltage is abandoned. Especially, the voltage induction phenomenon is influenced very much by an inrush current when electric power is put into power line. The magnetic flux can double depending on input phase even if there is no remanent flux, and it can be greater if there is remanent flux. Furthermore, since the cable does not consist of a ferromagnetic material, the magnetic flux can be increased by twice, raising the induced voltage of signal line. Such phenomena can be explained as the voltage measured on control signal line changes when the power is on and off, and the result of this test is a comprehensive result of these phenomena.

\subsection{Characteristics of electromagnetic field and composition of simulation modeling and circuit}

The cable used in the simulation was modeled in the actual size to raise the reliability of simulation result by referring to the specifications of $0.6 / 1 \mathrm{kV}$ TFR-CVVS, $8 \mathrm{P} \times 1.5 \mathrm{~mm}^{2}$ cable (hereinafter integrated cable) of LS electric wire. Figs. 8 and 9 are respectively the cross section and model of TFR-CVVS cable referred to for modeling and Table 2 shows the materials of cable composition. Also, the simulation must copy and reflect the environment and dynamic characteristics of test environment as much as possible. By assuming the power voltage used in the induced voltage measurement experiment as the induced
Table 2. Materials constituting TFR-CVVS Cable [8]

\begin{tabular}{c|c|c}
\hline Items & Construction & Material or type \\
\hline$* 1$ & Conductor & Circular stranded soft wire \\
\hline 2 & insulator & Vinyl (PVC) \\
\hline 3 & interposition & Proper interposition \\
\hline 4 & Binders & Proper tape \\
\hline 5 & Ground wire & Tinned Soft copper wire \\
\hline 6 & Shielding & AI/mylar tape \\
\hline 7 & Sheath & Fire retardant vinyl (FR-PVC) \\
\hline
\end{tabular}

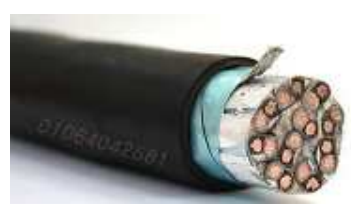

Fig. 8. Cross-sectional Diagram of TFR-CVVS Cable

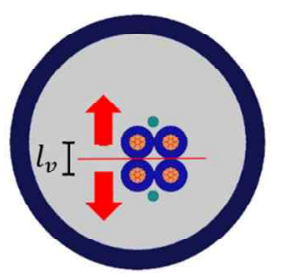

(a)

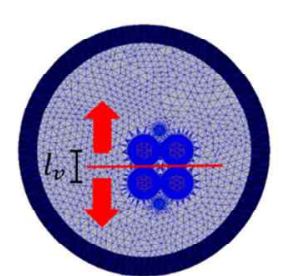

(b)
Fig. 9. 2D cable modelling: (a) 2D model; (b) Mesh (Finite Elements Method)

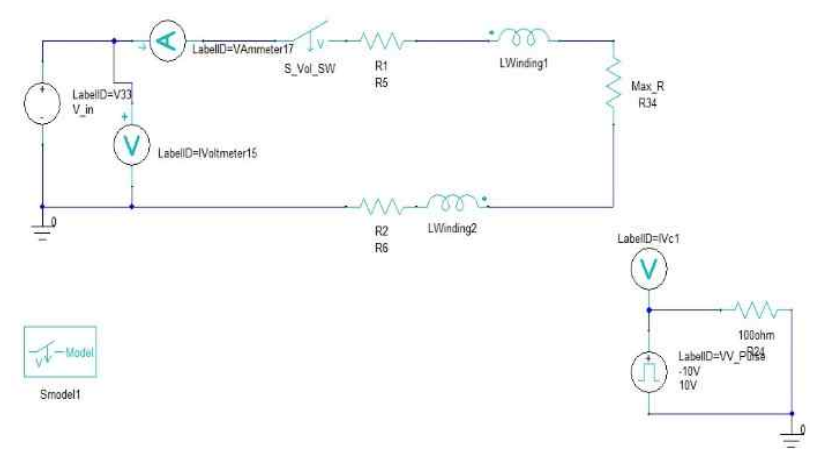

Fig. 10. Simulation circuit diagram

voltage of control signal line, this simulation constructed the simulation circuit as in Fig. 10 to artificially permit surge voltage to the power line at switch on/off to copy this in the simulation.

To estimate the voltage induced to the control signal line near the power line, the distance between the control signal line and power line of the cable modeling in Fig. 9 was determined as a variable (lv). Then, a simulation was conducted for the induced voltage generated by each cable separate from the power line to be similar to the experiment. This compared the size of induced voltage according to the separation distance in the same surge voltage. Since the integrated cable consists of a nonmagnetic material, an insulator, as in Table 2, the simulation method of separating the control signal line 


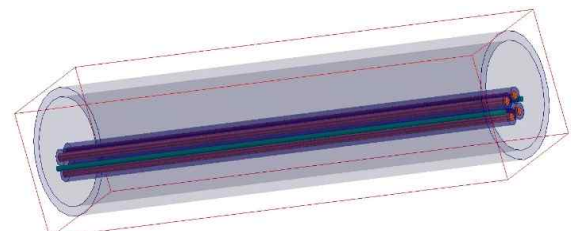

Fig. 11. 3D cable model

from the integrated cable can be seen as reasonable.

The surveyed cable resistance consisted of a control signal line that had 7 wires of cables in pairs at average of $4.25 \Omega$, the resistance of cable for the measurement of induced voltage was approximated at about $8.5 \Omega$ to be applied on the simulation. Also, the model used in the simulation was created in 2D as in Fig. 9 instead of 3D as in Fig. 11. This simulation method could be used because electromagnetic characteristics in a structure consisting of a cylindrical shape like a cable had a longitudinal axis and the same characteristics. Thus, the result of $2 \mathrm{D}$ simulation using the concept of infinite could be multiplied by the component of longitudinal axis to draw a result value. This has a very high reliability because it is the same as the electromagnetic simulation method of cylindrical motor. Furthermore, the higher the number of elements is, the higher the accuracy of simulation becomes when the finite element method is applied, so the reliability of simulation was obtained by increasing the mesh of 2D model.

\subsection{Analysis and review of simulation result}

As seen on Fig. 12, the analysis of induced voltage size while changing the surge voltage showed that the induced voltage of control signal line was proportional to the voltage size of surge. The surge voltage could exceed the rating of measuring equipment during the experiment, so the surge electric current value was assumed as the standard. The analysis also demonstrated through the simulation that if a pulse voltage to copy $800 \mathrm{~V}$ was permitted, about $17.2 \mathrm{~V}$ of induced voltage was abandoned to the control signal line. On the legend of the graph, the unit representing the size of surge voltage is $\mathrm{V}$.

The result value of this simulation was quite higher than the experiment value. However, the fact that a higher induced voltage of the control signal line could be generated in the simulation compared to the physical situation of actual cable indicates that the induced voltage was simulated in the worst stress condition. Moreover, since the size of magnetic flux linkage among cables varied depending on the separation distance between the power line and control signal line, the change of induced voltage was simulated by having the separation distance as a variable. The simulation also confirmed that the induced voltage of control signal line changed even when the distance between cables was changed based on a certain surge voltage. As mentioned previously, the magnetic flux due to the surge voltage permitted to the power line

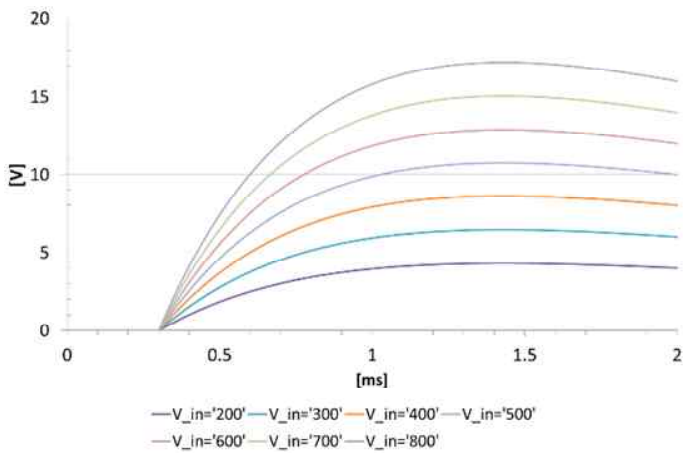

Fig. 12. Change of induced voltage by surge voltage size

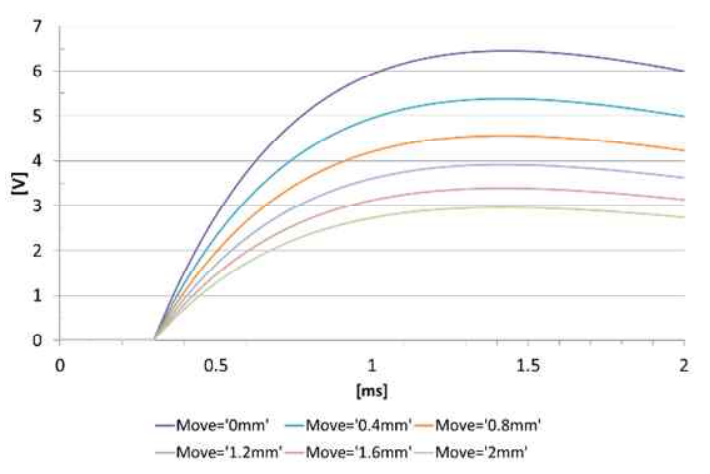

Fig. 13. Change of induced voltage according to the separation distance of power line and control signal line

influenced the magnetic flux that was linked to the control signal line, the amount of magnetic flux linkage decreased as the separation distance increased and the induced voltage also decreased due to the increase of leakage flux. Fig. 13 shows the result of induced voltage simulation that changed the separation distance from $0 \mathrm{~mm}$ to $2 \mathrm{~mm}$ based on $400 \mathrm{~V}$ of surge voltage. As seen on the result, the closer the distance was, the higher the induced voltage was generated.

\section{Conclusion}

Based on the above simulation result, induced voltage was likely to occur among neighboring cables due to the voltage change of a nearby cable and voltage permitted by surge. Also, the simulation result confirmed that the size of voltage induced by a separation distance among cables and the size of surge voltage. This result demonstrated that the cables used as major signal lines in control must secure a power line and enough separation distance to generate a huge magnetic field nearby.

\section{Acknowledgements}

This work was supported by the National Research 
Foundation of Korea (NRF) grant funded by the Korea government (MSIP) (No.2015R1C1A1A01055013).

This work was supported by the National Research Foundation of Korea (NRF) grant funded by the Korea government (MSIP) (No. 2013R1A2A1A01015171)

\section{References}

[1] Hyung-Woo Lee, Ki-Doek Lee, Mi-Jung Kim, JaeJun Lee, Jung-Ho Han, Tae-Chul Jeong, Ho-Joon Lee, Gwang-Hyeon Ryu, Hyun-Jong Park and Ju Lee, "Performance Analysis of a PM Motor by Changing the Magnitude and the Path of Flux," Journal of the Korean Physical Society, vol.62, no.1, pp.136-141, Jan. 2013.

[2] Chan-Bae Park, Hyung-Woo Lee, and Ju Lee, "Performance Analysis of the Linear Induction Motor for the Deep-Underground High-Speed GTX," Journal of Electrical Engineering \& Technology, vol. 7, no. 2, pp. 200-206, Mar.2012.

[3] Han Ke, Wei-Jen Lee, Mo-Shing Chen, Jih-Phong Liu, Jin Shyr Yang, "Grounding Techniques and Induced Surge Voltage on the Control Signal Cables," IEEE Trans. Industry Applications, vol. 34, no. 4, pp. 663-668, Aug. 1998

[4] Hossien Heydari, Vahid Abbasi, Faramarz Faghihi, "Impact of Switching-Induced Electromagnetic Interference on Low-Voltage Cables in Substations", IEEE Trans. Electromagnetic Compatibility, vol. 51, no. 4, pp. 937-944, Nov. 2009

[5] Hyung-Woo Lee, Chan-Bae Park and Ju Lee, "Improvement of Thrust Force Properties of Linear Synchronous Motor for an Ultra-High-Speed Tube Train," IEEE Trans. Magn., vol. 47, no. 11, pp. 46294634, Nov.2011.

[6] L. O. Chua, M. Hasler, J. Neiyrinck, and P. Verbugh, "Dynamics of a piecewise-linear resonant circuit," IEEE Trans. Circuits Syst., vol. CAS-29, pp. 1057c1061, Aug. 1982.

[7] Ju Lee, Electric Machinery: Hongrung Publishing, 2011, p.99-120.

[8] IEC/TR 61000-5-2 Ed. 1.0 Electromagnetic compatibility (EMC) - Part 5: Installation and mitigation guidelines - Section 2: Earthing and cabling

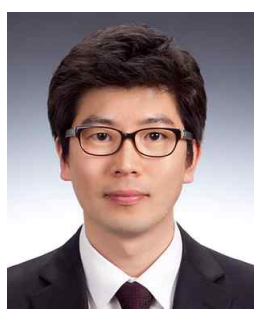

Dong-Woo Kang received his B.S., M.S. and Ph.D. degrees in Electrical Engineering from Hanyang University, Seoul, Korea in 2006, 2008 and 2011 respectively. He joined Keimyung University is currently a Professor of the Dept. of Electrical Energy Engineering. His research interests include design, analysis, of motor/generator; power conversion systems; and applications of spherical motor for robots; home appliances

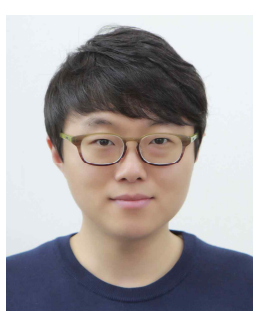

In-Gun Kim received his B.S. degree in Electrical Engineering from Incheon University, Incheon, Korea in 2011, and his M.S. degree in Electrical Engineering from Hanyang University, Seoul, Korea in 2013. Since 2013, he has been pursuing the Ph.D. degree at the Department of Electrical Engineering, Hanyang University. His research interests include design, analysis, testing and control of motor/generator; power conversion systems; and applications of motor drive, such as electric vehicles.

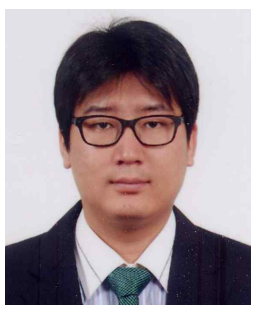

Sang-Hwan Ham received his B.S., M.S. and Ph.D. degrees in Electrical Engineering from Hanyang University, Seoul, Korea in 2006, 2008 and 2011 respectively. He joined Kyungil University and is currently a Professor of the school of Electrical and Railway Engineering. His research interests include design, analysis, of motor/generator.

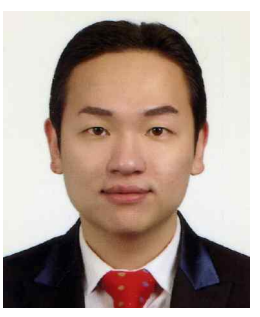

Sung-Yul Kim received the B.S. and Ph.D. degrees in electrical engineering from Hanyang University, Seoul, Korea, in 2007 and 2012. From 2012 to 2013, he was a research assistant at Georgia Institute of Technology, GA, USA. Since 2013, he has been with the Department of Electrical Energy Engineering, Keimyung University, Daegu, Korea. His main research interests include computer aided optimization, renewable energy sources applied to mircrogrid, and power system reliability.

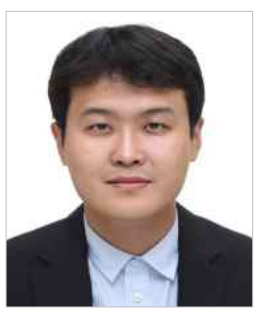

Sungwoo Bae received the B.S. degree from Hanyang University, Seoul, Korea, and the M.S.E. and Ph.D. degrees from the University of Texas at Austin, USA, all in electrical engineering, in 2006, 2009, and 2011, respectively. From 2012 to 2013, he was a senior research engineer with Power Center at Samsung Advanced Institute of Technology. He has been an Assistant Professor in the department of electrical engineering at Yeungnam University in Korea since 2013. In 2005, Dr. Bae was awarded the Grand Prize at the national electrical 
engineering design contest by the Minister of Commerce, Industry and Energy of the Republic of Korea.

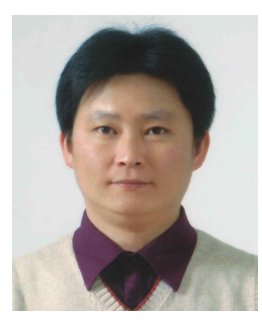

Dae-Nyeon Kim has graduated and obtained the B.S. and M.S. from Control and Instrumentation Eng. of University of Ulsan, Korea, in 2001 and 2003, respectively. He has obtained the Ph.D. from Electrical Engineering Department of University of Ulsan, Korea, in 2009. He has worked in University of Ulsan(Electrical Engineering Department) as a visiting Professor during 2009 to 2010. During 2010 (March)-2012 (August), he has worked as a Professor, director of Energy Harvesting Systems Lab., Dept. of New and Renewable Energy Engineering, Kundong University. $\mathrm{He}$ has served as the vice dean of Dept. of Engineering during 2010 to 2012. Since September 2012, he has joined and now as a Researcher of Research and Development, Team Leader of Research Equipment Technology Team, Kyeongbuk Institute of IT Convergence Industry Technology. His research interests include embeded system, energy harvesting and intelligent system.

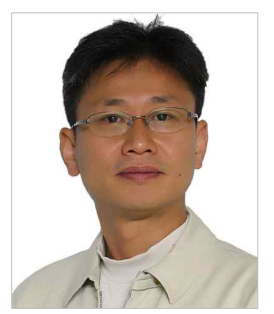

Ju Lee He received his M.S. degree from Hanyang University, Seoul, South Korea, in 1988, and his Ph.D. from Kyusyu University, Japan in 1997, both in Electrical Engineering, $\mathrm{He}$ joined Hanyang University in September, 1997 and is currently a Professor of the Division of Electrical and Biomedical Engineering. His main research interests include electric machinery and its drives, electromagnetic field analysis, new transformation systems such as hybrid electric vehicles (HEV), and high-speed electric trains and standardization. He is a member of the IEEE Industry Applications Society, Magnetics Society, and Power Electronics Society. 